\title{
Des microARN au cour de la fabrication de cils motiles
}

\author{
Olivier Mercey ${ }^{1,2}$, Benoît Chevalier ${ }^{1,2}$, Laurent Kodjabachian ${ }^{3}$,
} Pascal Barbry ${ }^{1,2}$, Brice Marcet ${ }^{1,2}$

$>$ Le cil, organite cellulaire conservé chez de nombreux eucaryotes, est un prolongement membranaire constitué de microtubules et doté de fonctions sensorielles et/ou locomotrices particulières [1]. On distingue le cil primaire, non-motile et présent dans la plupart des types cellulaires, du cil motile qui est présent en un unique exemplaire dans des cellules «monociliées », et jusqu'à plusieurs centaines dans des cellules dites «multiciliées » $(\rightarrow)$. Chez les vertébrés, ces cellules multiciliées bordent la surface de certains tissus comme les voies respiratoires, les ventri- $(\rightarrow)$ Voir le numéro thématique «Cils primaires $m / s n^{\circ} 11$, novembre 2014 et ciliopathies », cules cérébraux, le tractus génital féminin, les placodes ${ }^{1}$ olfactives du poisson zèbre ou encore l'épiderme d'embryon de grenouille [2, 3]. Grâce au battement coordonné des cils motiles, les cellules multiciliées jouent un rôle important dans divers processus physiologiques comme l'évacuation, par l'épithélium respiratoire, des particules inhalées piégées dans le mucus, la circulation du liquide céphalo-rachidien ou la progression de l'embryon dans le tractus génital [2]. Un dysfonctionnement ou une diminution du nombre de ces cils motiles peut être la cause ou aggraver les symptômes de nombreuses pathologies telles que des ciliopathies (comme les dyskinésies ciliaires touchant le système respiratoire) ou des maladies respiratoires chroniques (comme la mucoviscidose,

${ }^{1}$ Ébauches de l'épithélium olfactif au cours du développement embryonnaire. l'asthme ou les broncho-pneumopathies chroniques obstructives) [2]. La fabrication de multiples cils motiles par les cellules multiciliées (processus appelé multiciliogenèse) intervient au cours du développement embryonnaire, ainsi que lors de la régénération de certains épithéliums spécialisés. Elle nécessite plusieurs étapes clés successives: (1) la sortie du cycle cellulaire, (2) l'acquisition de l'identité «cellule multiciliée » sous le contrôle des voies de signalisation Notch et BMP (bone morphogenetic protein), (3) la réorganisation du réseau apical d'actine, (4) la multiplication massive de centrioles, qui vont migrer et s'ancrer dans le réseau apical d'actine pour devenir des corps basaux, et enfin (5) l'élongation des axonèmes ${ }^{2}$ des cils motiles à partir de ces mêmes corps basaux qui jouent le rôle de centre organisateur des microtubules à la base de chaque cil (Figure 1) [4]. L'élucidation des mécanismes qui gouvernent la multiciliogénèse est un prélude indispensable à la mise en place de nouvelles stratégies thérapeutiques plus efficaces pour lutter contre les pathologies associées à des désordres ciliaires. Plusieurs facteurs de transcription, comme Foxll (Forkhead box protein $J 1$ ) ou certains membres de la famille RFX (regulatory factor $X$ ) [5], de même que les voies de signalisation Notch et BMP $[3,6]$, étaient déjà connus pour agir à plusieurs étapes clés de la multiciliogénèse. Notre équipe s'est concentrée sur l'étude du rôle potentiel des microARN dans ce mécanisme.

\footnotetext{
${ }^{2}$ Partie axiale motrice du cil.
}

${ }^{1}$ CNRS, institut de pharmacologie moléculaire et cellulaire, UMR7275, Sophia-Antipolis, F06560, France ;

${ }^{2}$ Université de Nice-Sophia-Antipolis, 660 , route des Lucioles, F-06560 Sophia-Antipolis, France

${ }^{3}$ Aix-Marseille Université, CNRS, UMR7288, institut de biologie du développement de Marseille, F13288 Marseille, France. barbry@ipmc.cnrs.fr marcet@ipmc.cnrs.fr

En 2011, nous avions établi un lien fonctionnel, conservé des amphibiens jusqu'à I'homme, entre la famille de microARN ( $m i A R N$ ou miR) miR-34/449 et la multiciliogénèse [3]. Les miARN sont des petits ARN non-codants endogènes qui contrôlent de nombreux processus biologiques en réprimant l'expression génique par des mécanismes post-transcriptionnels [7]. Nous avions montré, à partir d'épithélium respiratoire humain et d'épiderme d'embryon de xénope, que les miR-34/449, dont l'expression était fortement et spécifiquement induite dans les cellules multiciliées, déclenchaient la multiciliogénèse en bloquant à la fois le cycle cellulaire et la voie Notch, deux étapes précoces de la différenciation «multiciliée » [3]. Nos résultats ont depuis été confirmés par deux études montrant que des souris invalidées pour miR-34/449 présentent des défauts de multiciliogénèse $[8,9]$. Plus récemment, nous avons mis en évidence une boucle de régulation négative réciproque entre miR-34/449 et la voie Notch [10]. Cette boucle permet l'accumulation de molécules miR-34/449 dans les cellules multiciliées engagées dans leur voie de différenciation, offrant la possibilité à ces microARN de contrôler d'autres cibles à des étapes plus tardives de la multiciliogénèse. En accord avec cette hypothèse, les travaux de Song et ses collègues suggèrent que la protéine centriolaire Cpl10, dont l'expression doit être réprimée pour permettre la maturation des corps basaux, représenterait une cible supplémentaire importante des miR-34/449 [8]. 


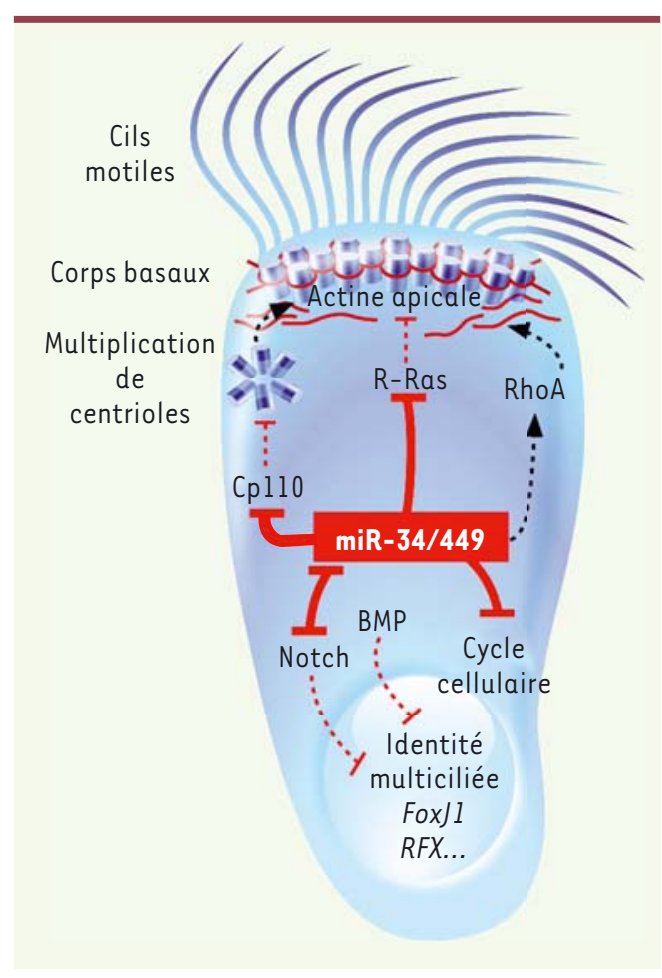

Figure 1. Illustration du rôle des miR-34/449 au cours de la multiciliogenèse. L'inhibition des voies BMP (bone morphogenetic protein) et Notch est un événement précoce requis pour l'entrée en différenciation «multiciliée ». L'expression spécifique des miR-34/449 dans les progéniteurs des cellules multiciliées permet la sortie du cycle cellulaire et l'initiation de l'acquisition de l'identité « multiciliée » en inhibant la voie Notch. L'inhibition de la voie Notch renforce l'expression de miR-449. Les miR-34/449 favorisent ensuite (1) la réorganisation du réseau apical d'actine, en réprimant $R$-Ras et en modulant l'activité de RhoA, et (2) la multiplication massive et la maturation des corps basaux, en réprimant $\mathrm{Cp} 110$. Après leur migration au pôle apical, ces corps basaux s'ancrent au réseau apical d'actine. En parallèle, les facteurs de transcription FoxJl (Forkhead box protein J1) et RFX (regulatory factor X) activent l'expression des constituants nécessaires à l'assemblage et au mouvement des cils motiles.

voie RhoA et indépendantes de la voie Notch, dans le contrôle de la réorganisation apicale du réseau d'actine qui s'opère lors de la multiciliogenèse.

Nous avons ainsi identifié, parmi les cibles des miR-34/449, la petite protéine G R-Ras (Rasrelated protein) comme une des cibles, conservées de l'amphi-

Nous montrons aujourd'hui, dans une nouvelle étude, que ces mêmes miR34/449 contrôlent également la formation du réseau apical d'actine, une autre étape clé de la multiciliogenèse, en agissant sur la voie des petites protéines G [10]. L'invalidation spécifique des miR-34/449 (à l'aide d'oligonucléotides antisens), aussi bien dans l'épiderme d'embryon de xénope que dans l'épithélium respiratoire humain, perturbe fortement la formation du réseau apical d'actine et des cils motiles. Dans les cellules respiratoires humaines, I'expression des miR-34/449 favorise la formation des fibres de stress d'actine et des points focaux d'adhésion. Elle induit également une augmentation de l'activité de la petite protéine G RhoA (Ras homolog family member $A$ ), régulateur clé du cytosquelette d'actine, et ce d'une manière indépendante de la voie Notch (Figure 1). Chez le xénope, l'inhibition des miR-34/449 bloque la réorganisation apicale du réseau d'actine et la multiciliogenèse, tandis que l'activité RhoA est toujours détectable. Ces résultats suggèrent l'implication d'autres cibles des miR-34/449, en aval de la bien à l'homme, contribuant à la formation du réseau apical d'actine au cours de la multiciliogenèse. Dans ces deux espèces, les miR-34/449 répriment spécifiquement l'expression du gène RRAS, à la fois au niveau du transcrit et de la protéine (Figure 1) [10].

Il est à noter que, chez l'homme, une invalidation trop précoce du gène RRAS à l'aide d'une stratégie d'interférence utilisant un siARN (small interfering RNA) affecte le remodelage apical d'actine et la multiciliogénèse, tout en augmentant l'activité RhoA, suggérant que R-Ras est probablement nécessaire aux premiers stades de la multiciliogenèse. De plus, dans nos deux modèles, le blocage spécifique de la liaison des miR-34/449 aux transcrits du gène RRAS réprime à la fois la réorganisation du réseau apical d'actine et la multiciliogenèse, mimant ainsi les effets obtenus par l'invalidation des miR-34/449. Dans les embryons de xénope, ces défauts sont corrigés lorsque l'on empêche les transcrits du gène RRAS, protégés contre I'action des miR-34/449, d'être traduits. Enfin, l'action de miR-34/449 aboutit à une relocalisation de la filamine-A, une protéine de liaison à l'actine, à proximité des corps basaux, et à la stabilisation du réseau apical d'actine, pré-requis indispensable pour l'ancrage des corps basaux. L'ensemble de nos résultats indiquent que les miR-34/449 contrôlent la réorganisation du réseau apical d'actine au cours de la multiciliogenèse en réprimant directement le gène RRAS, d'une manière indépendante de la voie Notch [10].

En conclusion, nos travaux illustrent comment certains microARN sont capables de contrôler les étapes successives d'un processus biologique complexe, via la répression de plusieurs molécules clés de voies de signalisation distinctes. Ce travail ouvre de nouvelles perspectives pour l'étude de l'étiologie des ciliopathies et offre également un éclairage intéressant en cancérologie, puisque l'amplification du nombre de centrioles est une caractéristique fréquente des cellules tumorales. $\diamond$

MicroRNAs pull the strings

of motile cilia

\section{LIENS D'INTÉRÊT}

Les auteurs déclarent n'avoir aucun lien d'intérêt concernant les données publiées dans cet article. Ce travail a fait l'objet d'un brevet CNRS n0 09/03723.

\section{RÉFÉRENCES}

1. Satir P, Guerra C, Bell AJ. Evolution and persistence of the cilium. Cell Motil Cytoskeleton 2007 ; 64 : 906-13.

2. Fliegauf M, Benzing T, Omran H. When cilia go bad: cilia defects and ciliopathies. Nat Rev Mol Cell Biol $2007 ; 8: 880-93$.

3. Marcet B, Chevalier B, Luxardi G, et al. Control of vertebrate multiciliogenesis by miR-449 through direct repression of the Delta/Notch pathway. Nat Cell Biol $2011 ; 13: 693-9$. 


\section{RÉFÉRENCES}

4. Vladar EK, Stearns T. Molecular characterization of centriole assembly in ciliated epithelial cells. J Cell Biol 2007 ; 178 : 31-42.

5. Thomas J, Morlé L, Soulavie F, et al. Transcriptional control of genes involved in ciliogenesis: a first step in making cilia. Biol Cell $2010 ; 102: 499-513$.

6. Cibois M, Luxardi G, Chevalier B, et al. BMP signalling controls the construction of vertebrate mucociliary epithelia. Development 2015 ; 142 : 2352-63.
7. Giovannini-Chami L, Grandvaux N, Zaragosi LE, et al. Impact of microRNA in normal and pathological respiratory epithelia. Methods Mol Biol 2011 ; 741 : 171-91.

8. Song R, Walentek P, Sponer N, et al. miR-34/449 miRNAs are required for motile ciliogenesis by repressing cpl10. Nature 2014 ; 510 : 115-20.

9. Wu J, Bao J, Kim M, et al. Two miRNA clusters, miR$34 \mathrm{~b} / \mathrm{c}$ and miR-449, are essential for normal brain development, motile ciliogenesis, and spermatogenesis. Proc Natl Acad Sci USA 2014 ; 111 : ع2851-7.
10. Chevalier B, Adamiok A, Mercey 0, et al. miR-34/449 control apical actin network formation during multiciliogenesis through small GTPase pathways. Nat Commun $2015 ; 6: 8386$

\section{NOUVELLE}

\section{Les macrophages}

\section{Nouveaux modulateurs de la répartition de la masse grasse au cours de l'obésité}

Élise Dalmas ${ }^{1}$, Nicolas Venteclef ${ }^{2}$

\begin{abstract}
${ }^{1}$ Département d'endocrinologie, diabète et métabolisme, centre hospitalier de l'université de Bâle, département de biomédecine, université de Bâle, $\mathrm{CH}-4031$ Bâle, Suisse. 2 Inserm, UMR S 113, Sorbonne Universités, UPMC Paris 6, université Paris Descartes, Sorbonne Paris Cité, centre de recherche des Cordeliers, 15, rue de l'École de Médecine, F-75006 Paris, France.

nicolas.venteclef@upmc.fr
\end{abstract}

> L'obésité et ses complications, telles que le diabète de type 2 ou l'athérosclérose, sont aujourd'hui reconnues comme des états inflammatoires chroniques de bas bruit, c'est-à-dire évoluant de manière relativement silencieuse (sans symptômes). Le tissu adipeux, principal lieu de stockage de l'excès d'apports caloriques, est au cœur de cette activité inflammatoire due à une production accrue de facteurs délétères de type cytokines et chimiokines [1]. En 2003, deux équipes américaines ont décrit pour la première fois l'infiltration de cellules immunitaires, les macrophages, dans le tissu adipeux de souris obèses et chez l'homme. Cette infiltration de macrophages semble être proportionnelle à l'indice de masse corporelle chez les sujets en surpoids et obèses [2, 3]. Ces macrophages, s'accumulant typiquement autour d'adipocytes hypertrophiques, sont considérés comme la source majeure de production de facteurs pro-inflammatoires tels que le TNF- $\alpha$ (tumor necrosis factor-alpha) ou l'interleukine(IL)-1 $\beta$. La production d'IL-1 $\beta$ favoriserait le maintien d'un dialogue pro-inflammatoire et prodiabétogène avec des lymphocytes Thelper de type 17 (Th17) au sein du tissu adipeux viscéral de patients obèses [4]. Ainsi, en plus de leur accumulation, les macrophages orchestreraient un dialogue entre les différentes cellules immunitaires du tissu adipeux, déterminant dans le développement de l'obésité et du diabète de type 2.

Les macrophages du tissu adipeux sont au cœur d'une inflammation prodiabétogène

Dans les modèles murins, l'abondance des macrophages dans le tissu adipeux peut être contrôlée par des manipulations génétiques ou des interventions pharmacologiques. Ces approches permettent de démontrer l'importance de ces cellules dans le développement des complications liées à l'obésité [1]. Chez l'homme, la présence de macrophages et la sécrétion de cytokines proinflammatoires semblent également associées à l'obésité et au diabète de type $2[1,4]$. Ces études restent cependant corrélatives. Sur le plan clinique, l'inflammation et la présence de macrophages sont particulièrement observées dans le tissu adipeux viscéral (intra-abdominal) et, de manière moins importante, dans la graisse sous-cutanée dite superficielle. L'élargissement du tissu adipeux viscéral (phénomène qualifié d'obésité androïde) est fortement associé à un risque élevé de développer un syndrome métabolique et des complications cardiovasculaires [5]. Au contraire, l'obésité gynoïde (accumulation préférentielle de la graisse dans les régions superficielles) est associée à un meilleur profil métabolique. Au cours de nos travaux, nous avons examiné le rôle possible des macrophages dans le développement soit de l'obésité gynoïde (protectrice), soit de l'obésité androïde (délétère).

Il existe de nombreux niveaux d'activation des macrophages dans les pathologies. Une nomenclature restrictive permet cependant de distinguer deux grandes classes de macrophages inflammatoires dites polarisations ${ }^{1} \mathrm{Ml}$ et M2: (1) les macrophages dits Ml, activés en particulier par le lipopolysaccharide $^{2}$ (LPS) via les récepteurs TLR (toll like receptors), suivent une voie classique aboutissant à la production

\footnotetext{
${ }^{1}$ La polarisation de cellules immunitaires, comme les macrophages, définit leur état d'activation qui dépend des cytokines qui les stimulent, des cytokines et autres facteurs qu'elles sécrètent, et des facteurs de transcription qu'elles expriment.

${ }^{2}$ Le lipopolysaccharide est une molécule présente dans la
} membrane externe des bactéries à Gram négatif. 